\title{
REGULAR TRANSITION FUNCTIONS AND REGULAR SUPERPROCESSES
}

\author{
E. B. DYNKIN
}

\begin{abstract}
The class of regular Markov processes is very close to the class of right processes studied by Meyer, Getoor and others. We say that a transition function $p$ is regular if it is the transition function of a well-defined regular Markov process. A characterization of regular transition functions is given which implies that, if $p$ is regular, then the Dawson-Watanabe and the FlemingViot supertransition functions over $p$ belong to the same class.
\end{abstract}

\section{INTRODUCTION}

1.1. Let $\left(E_{t}, \mathscr{B}_{t}\right), t \in \mathbf{R}$ be a family of measurable spaces. A transition function $p$ in $\left(E_{t}, \mathscr{B}_{t}\right)$ is a function $p(r, x ; t, B), r<t \in \mathbf{R}, x \in E_{r}, B \in \mathscr{B}_{t}$ with the following properties:

1.1.A. $p(r, x ; t, \cdot)$ is a probability measure on $\left(E_{t}, \mathscr{B}_{t}\right)$.

1.1.B. $p(r, \cdot ; t, B)$ is a $\mathscr{B}_{r}$-measurable function.

1.1.C. $\int p(r, x ; s, d y) p(s, y ; t, B)=p(r, x ; t, B)$ for all $r<t, x$ and $B$.

We say that $p$ separates states if:

1.1.D. For every $\alpha \in \mathbf{R}$ and every $x \neq y \in E_{\alpha}$, there exist $t>\alpha$ and $B \in \mathscr{B}_{t}$ such that $p(\alpha, x ; t, B) \neq p(\alpha, y ; t, B)$.

We set $p(r, x ; t, B)=0$ for $r \geq t$. We denote by $\mathscr{E}$ the set of all pairs $(t, x), t \in \mathbf{R}, x \in E_{t}$ and by $\mathscr{B}_{\mathscr{E}}$ the $\sigma$-algebra in $\mathscr{E}$ generated by the functions

$$
F(r, x)=p(r, x ; t, B), \quad t \in \mathbf{R}, B \in \mathscr{B}_{t} .
$$

If all spaces $\left(E_{t}, \mathscr{B}_{t}\right)$ are standard Borel, then $p$ is called $a$ Borel transition function.

1.2. Suppose that we are given:

(a) an arbitrary set $\Omega$;

(b) for every $t \in \mathbf{R}$, a measurable space $\left(E_{t}, \mathscr{B}_{t}\right)$ and a mapping $X_{t}$ from $\Omega$ to $E_{t}$;

Received by the editors May 5, 1988 .

1980 Mathematics Subject Classification (1985 Revision). Primary 60J25, 60J50, 60J57, 60G60, 60G35; Secondary 60J70, 60J80.

Key words and phrases. Right processes, regularization of a Markov process, entrance laws, superprocesses, measure-valued Markov processes.

Partially supported by National Science Foundation Grant DMS- 8505020 and by the U.S. Army Research Office through the Mathematical Sciences Institute at Cornell University. 
(c) for every open interval $\Delta$, a $\sigma$-algebra $\mathscr{F}(\Delta)$ in $\Omega$;

(d) for every $r \in \mathbf{R}, x \in E_{r}$, a probability measure $P_{r, x}$ on $\mathscr{F}_{>r}=$ $\mathscr{F}(r, \infty)$.

We assume that $\mathscr{F}(\Delta) \subset \mathscr{F}\left(\Delta^{\prime}\right)$ for $\Delta \subset \Delta^{\prime}$ and that $\mathscr{F}(\Delta)$ is generated by $\bigcup \mathscr{F}\left(\Delta_{n}\right)$ if $\Delta_{n} \uparrow \Delta$. We denote by $\mathscr{F}_{\leq t}$ the minimal $\sigma$-algebra which contains $\mathscr{F}_{<t}$ and $\left\{X_{t} \in B\right\}$ for all $B \in \mathscr{B}_{t}$. The notation $\mathscr{F}_{>t}, \mathscr{F}(r, t], \ldots$ is defined similarly. We say that $\mathscr{X}=\left(X_{t}, \mathscr{F}(\Delta), P_{r, x}\right)$ is a Markov process if:

1.2.A. $X_{t}$ is adapted to $\mathscr{F}(\Delta)$ (i.e. $\left\{X_{t} \in B\right\} \in \mathscr{F}(\Delta)$ if $t \in \Delta$ and $\left.B \in \mathscr{B}_{t}\right)$.

1.2.B. For each $Y \in \mathscr{F}_{>t}, P_{t, x} Y$ is $\mathscr{B}_{t}$-measurable. For every $r<t \in \mathbf{R}$ and every $Y \in \mathscr{F}(r, t], Z \in \mathscr{F}_{>t}$,

$$
P_{r, x} Y Z=P_{r, x}\left(Y P_{t, X_{t}} Z\right) \text {. }
$$

[Writing $Y \in \mathscr{F}$ means that $Y$ is a bounded $\mathscr{F}$-measurable function.]

To every Markov process $\mathscr{X}$ there corresponds a transition function

$$
p(r, x ; t, B)=P_{r, x}\left\{X_{t} \in B\right\}, \quad r<t \in \mathbf{R}, x \in E_{r}, B \in \mathscr{B}_{t} .
$$

Every Borel transition function corresponds to a canonical Markov process $\mathscr{X}$ for which $\Omega$ is the space of all paths $\omega(t) \in E_{t}, t \in \mathbf{R}$ and $\mathscr{F}(\Delta)$ is generated by $X_{t}, t \in \Delta$.

1.3. If $\mathscr{X}$ is a Markov process, then we denote by $\mathscr{K}_{\alpha}(\mathscr{X})$ the set of all finite measures $P$ on $\mathscr{F}_{>\alpha}$ such that

$$
P Y Z=P\left(Y P_{t, X_{t}} Z\right)
$$

for all $\alpha<t, Y \in \mathscr{F}(\alpha, t], Z \in \mathscr{F}_{>t}$. Put $P \in \mathscr{K}_{\alpha}^{1}(\mathscr{X})$ if $P \in \mathscr{K}_{\alpha}(\mathscr{X})$ and $P(\Omega)=1$.

Denote by $\mathscr{K}_{\alpha e}^{1}(\mathscr{Z})$ the set of all extremal elements of $\mathscr{K}_{\alpha}^{1}(\mathscr{X})$ (that is the elements which can not be represented as a convex combination of two distinct elements) and by $\mathscr{N}_{\alpha}(\mathscr{X})$ the set of all measures $P_{\alpha, x}, x \in E_{\alpha}$.

We say that $\mathscr{X}$ is a regular process if:

1.3.A. For every $r<u \in \mathbf{R}$, every $Z \in \mathscr{F}_{>u}$ and every $P \in \mathscr{K}_{r}^{1}(\mathscr{X}), P_{t, X_{t}} Z$ is, $P$-a.s., right continuous in $t$ on $[r, u)$.

1.3.B. The $\sigma$-algebra $\mathscr{B}_{\mathscr{E}}$ is generated by a countable family $\mathscr{Q}$ of bounded functions $g^{t}(x)$ such that, for every $\omega \in \Omega, g^{t}\left(X_{t}(\omega)\right)$ is right continuous and has left limits at every $t \in \mathbf{R}$.

1.3.C. For all $r \in \mathbf{R}, x \in E_{r}, X_{r}=x \quad P_{r, x}$-a.s.

[If $P$ is a probability measure on $\mathscr{F}$, then the expression " $A$ holds $P$-a.s." means that $A$ belongs to the $P$-completion of $\mathscr{F}$ and $P(A)=1$.]

By replacing $\mathscr{K}_{r}^{1}(\mathscr{X})$ in condition 1.3.A with smaller class $\mathscr{N}_{r}(\mathscr{X})$, we define the slightly wider class of right processes.

1.4. Let $\mathbf{H}^{t}$ stand for the cone of all positive bounded $\mathscr{B}_{t}$-measurable functions, let $\mathscr{M}_{t}$ be the set of all finite measure on $\mathscr{B}_{t}$ and let $\mathscr{M}_{t}^{1}$ stand for the subset of $\mathscr{M}_{t}$ which consists of probability measures. Put $\langle f, \mu\rangle=\int f d \mu$. To every 
transition function $p$ in $\left(E_{t}, \mathscr{B}_{t}\right)$, there correspond linear operators $f \rightarrow T_{t}^{r} f$ from $\mathbf{H}^{t}$ to $\mathbf{H}^{r}$ and linear operators $\mu \rightarrow \mu T_{t}^{r}$ from $\mathscr{M}_{r}$ to $\mathscr{M}_{t}$ such that $\left\langle T_{t}^{r} f, \mu\right\rangle=\left\langle f, \mu T_{t}^{r}\right\rangle$.

An entrance law for $p$ at time $\alpha$ is a family $\kappa_{t} \in \mathscr{M}_{t}, t>\alpha$ such that $\kappa_{r} T_{t}^{r}=\kappa_{t}$ for all $\alpha<r<t$. We denote by $\mathscr{K}_{\alpha}(p)$ the set of all such laws, by $\mathscr{K}_{\alpha}^{1}(p)$ the set of all probability entrance laws (for which $\kappa_{t}$ are probability measures) and by $\mathscr{N}_{\alpha}(p)$ the set of the entrance laws of the form

$$
\kappa_{t}(B)=p(\alpha, x ; t, B), \quad t>\alpha, B \in \mathscr{B}_{t} .
$$

If $\mathscr{X}$ is a Markov process with the transition function $p$, then the formula

$$
\kappa_{t}(B)=P\left\{X_{t} \in B\right\}, \quad t \in \mathbf{R}, B \in \mathscr{B}_{t},
$$

determines a one-to-one mapping from $\mathscr{K}_{\alpha}(\mathscr{X})$ into $\mathscr{K}_{\alpha}(p)$ which maps $\mathscr{K}_{\alpha}^{1}(\mathscr{X})$ into $\mathscr{K}_{\alpha}^{1}(p)$ and $\mathscr{N}_{\alpha}(\mathscr{X})$ into $\mathscr{N}_{\alpha}(p)$. We say that $\mathscr{Z}$ is well defined if (1.5) is a one-to-one mapping from $\mathscr{K}_{\alpha}^{1}(\mathscr{X})$ onto $\mathscr{K}_{\alpha}^{1}(p)$. In particular, every canonical Markov process is well defined.

A transition function $p$ is called regular if it is the transition function of a well-defined regular process.

Theorem 1.1. Suppose that $p$ is a Borel transition function which separates states. Then $p$ is regular if and only if it satisfies the following two conditions:

1.4.A. The $\sigma$-algebra $\mathscr{B}_{\mathscr{E}}$ is generated by an algebra $\mathscr{G}$ of bounded functions $g$ such that, for every $r \in \mathbf{R}$ and for all $x \in E_{r}, T_{t}^{r} g^{t}(x) \rightarrow g^{r}(x)$ as $t \downarrow r$.

1.4.B. For every $\alpha \in \mathbf{R}, \mathscr{K}_{\alpha e}^{1}(p)=\mathscr{N}_{\alpha}(p)$.

1.5. Every regular transition function has the following property:

1.5.A. For every positive $\mathscr{B}_{\mathscr{E}}$-measurable $f^{t}(x)$, the function $T_{t}^{r} f^{t}(x)$ is $\mathscr{B}_{\mathscr{E}} \times \mathscr{B}_{\mathbf{R}}$-measurable in $(r, x), t$ (here $\mathscr{B}_{\mathbf{R}}$ means the Borel $\sigma$-algebra in $\mathbf{R}$ ).

Suppose that $p$ is a Borel transition function which satisfies 1.5.A. Then (see [D5]), for every constant $\gamma>0$, there exists a unique semigroup of operators $V_{t}^{r}: \mathbf{H}^{t} \rightarrow \mathbf{H}^{r}$ such that

$$
V_{t}^{r} f+\frac{\gamma}{2} \int_{r}^{t} T_{t}^{r}\left[\left(V_{t}^{s} f\right)^{2}\right] d s=T_{t}^{r} f
$$

for all $r<t \in \mathbf{R}, f \in \mathbf{H}^{t}$. Let $\mathscr{B}_{\mathscr{M}_{t}}$ be the $\sigma$-algebra in $\mathscr{M}_{t}$ generated by the functions $F(\nu)=\nu(B), B \in \mathscr{B}_{t}$. There exists a unique transition function $\mathscr{P}(r, \mu ; t, d \nu)$ in $\left(\mathscr{M}_{t}, \mathscr{B}_{\mathscr{M}_{t}}\right)$ such that

$$
\int_{\mathscr{M}_{t}} \mathscr{P}(r, \mu ; t, d \nu) e^{-\langle f, \nu\rangle}=\exp \left\{-\left\langle V_{t}^{r} f, \mu\right\rangle\right\}, \quad f \in \mathbf{H}^{t} .
$$

(see [D5], cf. [Da and W]). It follows from (1.7) that

$$
\int \mathscr{P}(r, \mu ; t, d \nu)\langle f, \nu\rangle=\left\langle T_{t}^{r} f, \mu\right\rangle .
$$

We call $\mathscr{P}$ the Dawson-Watanabe supertransition function over $p$. 
The Fleming-Viot supertransition function $\mathscr{P}$ over $p$ is defined in the spaces $\mathscr{M}_{t}^{1}$ by (1.8) and the inductive formula

$$
\begin{aligned}
\text { (1.9) } \int & \mathscr{P}(r, \mu ; t, d \nu) \prod_{i=1}^{n}\left\langle f_{i}, \nu\right\rangle=u_{n}(t-r) \prod_{i=1}^{n}\left\langle T_{t}^{r} f_{i}, \mu\right\rangle \\
& +2 \gamma \sum_{i<j} \int_{r}^{t} d s u_{n}(t-s) \int \mathscr{P}(r, \mu ; s, d \nu)\left\langle T_{t}^{s} f_{i} T_{t}^{s} f_{j}, \nu\right\rangle \prod_{k \neq i, j}\left\langle T_{t}^{s} f_{k}, \nu\right\rangle
\end{aligned}
$$

for $n \geq 2, f_{1}, \ldots, f_{n} \in \mathscr{H}^{t}$ where

$$
u_{n}(t)=e^{-\gamma n(n-1) t}
$$

(see [FV and DH], cf. [D6]).

Our central result is the following theorem.

Theorem 1.2. Suppose that a transition function $p$ separate states and let $\mathscr{P}$ be the Dawson-Watanabe or the Fleming-Viot supertransition function over $p$. If $p$ is regular, then $\mathscr{P}$ is also regular. If $\mathscr{P}$ is regular and if $p$ satisfies condition 1.4.A, then $p$ is also regular.

\section{Proof of Theorem 1.1}

2.1. Let $(E, \mathscr{B})$ and $(\tilde{E}, \tilde{\mathscr{B}})$ be two measurable spaces. Writing $(E, \mathscr{B}) \subset$ $(\tilde{E}, \tilde{\mathscr{B}})$ means that $E \in \tilde{\mathscr{B}}$ and $\mathscr{B}$ is the trace of $\tilde{\mathscr{B}}$ on $\tilde{E}$ (that is $\tilde{\mathscr{B}}$ consists of the sets $B \cap E, B \in \tilde{\mathscr{B}}$ ).

Suppose that $l$ is an isomorphism from $(E, \mathscr{B})$ into $(\tilde{E}, \tilde{\mathscr{B}})$ that is a oneto-one mapping from $E$ into $\tilde{E}$ such that $l(B) \in \tilde{\mathscr{B}}$ if and only if $B \in \mathscr{B}$. Then we can make $(E, \mathscr{B}) \subset(\tilde{E}, \tilde{\mathscr{B}})$ by identifying $l(x)$ with $x$.

We use the following facts:

2.1.A. If $l$ is a one-to-one measurable mapping from a standard Borel space $(E, \mathscr{B})$ into a standard Borel space $(\tilde{E}, \tilde{\mathscr{B}})$, then $l$ is an isomoprhism from $(E, \mathscr{B})$ into $(\tilde{E}, \tilde{\mathscr{B}})$.

2.1.B. Every countable family of measurable functions separating points of a standard Borel space $(E, \mathscr{B})$ generates $\mathscr{B}$.

We refer to [K, Section 39.5, Theorem 2] for 2.1.A and to [D2, Lemma 2.1] for 2.1.B (which follows easily from 2.1.A).

Lemma 2.1. Suppose that $p$ is a Borel transition function. Let $\Lambda$ be a countable everywhere dense subset of $\mathbf{R}$ and let a countable family $\mathscr{B}_{t}^{\prime} \subset \mathscr{B}_{t}$ be closed under intersection, contain $E_{t}$ and generate $\mathscr{B}_{t}$. Formula

$$
F(r, x)=p(r, x ; t, B), \quad t \in \Lambda, f \in \mathscr{B}_{t}^{\prime},
$$

determines a family $\mathscr{W}$ of functions on $\mathscr{E}$ which generates $\mathscr{B}_{\mathscr{C}}$.

If $p$ separates states, then for every $r$, the functions $(2.1)$ generate $\mathscr{B}_{r}$ and the mapping $x \rightarrow(r, x)$ is an isomorphism from $\left(E_{r}, \mathscr{B}_{r}\right)$ into $\left(\mathscr{E}, \mathscr{B}_{\mathscr{C}}\right)$.

Proof. Denote by $\mathscr{A}$ the $\sigma$-algebra in $\mathscr{E}$ generated by $\mathscr{W}$. Let $t \in \Lambda$. Put $f \in \mathscr{H}$ if $T_{t}^{r} f(x)$ is $\mathscr{A}$-measurable. By the Multiplicative Systems Theorem 
(see, e.g., [D5]), $\mathscr{H}$ contains all bounded $\mathscr{B}_{t}$-measurable functions. Now let $t$ be an arbitrary real number. Choose $t_{n} \in \Lambda$ such that $t_{n} \uparrow t$. Note that

$$
1_{r<t_{n}} T_{t}^{r} f(x)=1_{r<t_{n}} T_{t_{n}}^{r} f_{n}(x)
$$

where $f_{n}(x)=T_{t}^{t_{n}} f(x)$. Since $T_{t_{n}}^{r} f(x)$ and $1_{r<t_{n}}=T_{t_{n}}^{r} 1$ are $\mathscr{A}$-measurable, we establish that $T_{t}^{r} f(x)$ is $\mathscr{A}$-measurable by passing to the limit in (2.2).

The second part of the lemma will follow from 2.1.A, B if we show that the functions (2.1) with a fixed $r$ separate points of $E_{r}$. Suppose that

$$
p(r, x ; t, B)=p(r, y ; t, B)
$$

for all $B \in \mathscr{B}_{t}^{\prime}$.

Then by the Multiplicative Systems Theorem, $T_{t}^{r} f(x)=T_{t}^{r} f(y)$ for all $f \in$ $\mathscr{B}_{t}$. If this is true for all $t \in \Lambda$, then, by 1.1.C, it is true for an arbitrary $t>r$. Hence $x=y$.

2.2. Lemma 2.2. If $\mathscr{X}$ is a regular Markov process in Borel spaces $\left(E_{t}, \mathscr{B}_{t}\right)$, then:

2.2.A. For every $t \in \mathbf{R}, X_{t}$ is measurable relative to

$$
\mathscr{F}(t, t+)=\bigcap_{u>t} \mathscr{F}(t, u] .
$$

2.2.B. For all $\alpha \in \mathbf{R}, P \in \mathscr{K}_{\alpha}^{1}(\mathscr{X}), Z \in \mathscr{F}_{>\alpha}$,

$$
P Z=P P_{\alpha, X_{\alpha}} Z \text {. }
$$

Proof. By 1.3.B, $f^{t}\left(X_{t}\right) \in \mathscr{F}(t, t+)$ if $f \in \mathscr{Q}$ and therefore for all $f \in \mathscr{B}_{\mathscr{C}}$. In particular, $T_{u}^{t} f\left(X_{t}\right) \in \mathscr{F}(t, t+)$ for all $u>t, f \in \mathscr{B}_{u}$, and 2.2.A follows from Lemma 2.1 .

It is sufficient to check 2.2.B for $Z \in \mathscr{F}_{>u}, u>\alpha$. By (1.3), $P Z=P P_{t, X_{t}} Z$ for all $t \in(\alpha, u)$, and we get 2.2.B by passing to the limit as $t \downarrow \alpha$ and using 1.3.A.

2.3. Let $\mathscr{X}=\left(X_{t}, \mathscr{F}(\Delta), P_{r, x}\right)$ be a Markov process in standard Borel spaces $\left(E_{t}, \mathscr{B}_{t}\right)$. An $x \in E_{t}$ is called a branching point if $P_{t, x} \notin \mathscr{K}_{t e}^{1}(\mathscr{X})$. The set $E_{t}^{\circ}$ of all nonbranching points belongs to $\mathscr{B}_{t}$ and we denote by $\mathscr{B}_{t}^{\circ}$ the trace of $\mathscr{B}_{t}$ in $E_{t}^{\circ}$.

Theorem 2.1. Let a Borel transition function $p$ in $\left(E_{t}, \mathscr{B}_{t}\right)$ have no branching points and satisfy conditions 1.4.A and 1.1.D. Let $\mathscr{X}=\left(X_{t}, \mathscr{F}(\Delta), P_{r, x}\right)$ be the canonical Markov process corresponding to $p$. Then there exist standard Borel spaces $\left(\tilde{E}_{t}, \tilde{\mathscr{B}}_{t}\right) \supset\left(E_{t}, \mathscr{B}_{t}\right)$ and a Markov process $\left(\tilde{X}_{t}, \mathscr{F}(\Delta), \tilde{P}_{r, x}\right)$ in $\left(\tilde{E}_{t}, \tilde{\mathscr{B}}_{t}\right)$ with the transition function $\tilde{p}$ such that:

2.3.A. The set $\tilde{P}_{t, x}, x \in \tilde{E}_{t}$ coincides with $\mathscr{K}_{t e}^{1}(\mathscr{X})$.

2.3.B. $\tilde{X}$ is $\mathscr{F}(t, t+)$-measurable.

2.3.C. For $x \in E_{t}, \tilde{P}_{t, x}=P_{t, x}$ and $P_{t, x}\left\{\tilde{X}_{t}=x\right\}=1$. 
2.3.D. $P\left\{X_{t} \neq \tilde{X}_{t}\right\}=0$ for all $P \in \mathscr{K}_{r}(\mathscr{X}), r<t$.

2.3.E. $\tilde{p}(r, x ; t, B)=p\left(r, x ; t, B \cap E_{t}\right)$ for $r<t \in \mathbf{R}, x \in E_{r}, B \in \tilde{\mathscr{B}}_{t}$

2.3.F. If $\Lambda$ is a countable everywhere dense subset of $\mathbf{R}$, then, for all $r<u \in$ $\mathbf{R}, Y \in \mathscr{F}_{\geq u}, P \in \mathscr{K}_{r}^{1}(\mathscr{X})$,

$$
\tilde{P}_{t, \tilde{X}_{t}} Y=\lim _{s \downarrow t, s \in \Lambda} P_{s, X_{S}} Y \quad \text { for all } t \in[r, u) \quad P \text {-a.s. }
$$

and therefore $\tilde{P}_{t, \tilde{X}_{t}} Y$ is right continuous on $[r, u) P$-a.s. Moreover $\tilde{P}_{t, \tilde{X}_{t}} Y$ has left limits on $(r, u] \quad P$-a.s.

We call $\tilde{\mathscr{X}}$ the regularization of $\mathscr{X}$.

Proof. Put $\tilde{E}_{t}=\mathscr{K}_{t e}^{1}(\mathscr{X})$ and let $\tilde{\mathscr{B}}_{t}$ be the $\sigma$-algebra in $\tilde{E}_{t}$ generated by functions $F(P)=P f\left(X_{u}\right), u>t, f \in \mathscr{B}_{u}$. By Theorem 1.1 and $\S 3.6$ in [D3], there exists a Markov process $\tilde{\mathscr{X}}=\left(\tilde{X}_{t}, \mathscr{F}(\Delta), \tilde{P}_{t, x}\right)$ in $\left(\tilde{E}_{t}, \tilde{\mathscr{B}}_{t}\right)$ which satisfies 2.3.B and 2.3.F and such that

$$
\tilde{P}_{t, y}=P \quad \text { if } y=P \in \mathscr{K}_{t e}^{1}(\mathscr{X}) .
$$

Clearly, (2.5) implies 2.3.A.

Let $\chi: \mathscr{K}_{t e}^{1}(\mathscr{X}) \rightarrow \mathscr{K}_{t e}^{1}(p)$ and $l: E_{t} \rightarrow \mathscr{K}_{t e}^{1}(p)$ be the maps defined by (1.4) and (1.5) and let $\rho=\chi^{-1} l$. Note that $\rho(x)=P_{t, x}$ and, by (2.5), $\tilde{P}_{t, \rho(x)}=P_{t, x}$. Clearly, $\rho$ is a 1-1 measurable mapping from $E_{t}^{\circ}$ into $\tilde{E}_{t}$ and, by 2.1.A, we can identify $x$ and $\rho(x)$ and make $\left(E_{t}^{\circ}, \mathscr{B}_{t}^{\circ}\right) \subset\left(\tilde{E}_{t}, \tilde{\mathscr{B}}_{t}\right)$. Obviously, 2.3.C holds.

Let $g$ belong to the algebra $\mathscr{G}$ in 1.4.A. For every $r<t<u, P \in \mathscr{K}_{r}(\mathscr{X})$, $Y \in \mathscr{F}(r, t]$,

$$
P\left[Y P_{t, X_{t}} g^{u}\left(X_{u}\right)\right]=P\left[Y g^{u}\left(X_{u}\right)\right]=P\left[Y P_{t, \tilde{X}_{t}} g^{u}\left(X_{u}\right)\right] .
$$

[The first equation follows from (1.3) and the second one from 2.3.F and (1.3).] This implies

$$
P\left[Y T_{u}^{t} g^{u}\left(X_{t}\right)\right]=P\left[Y T_{u}^{t} g^{u}\left(\tilde{X}_{t}\right)\right]
$$

and passing to the limit as $u \downarrow t$, we get $P\left[Y g^{t}\left(X_{t}\right)\right]=P\left[Y g^{t}\left(\tilde{X}_{t}\right)\right]$. Since $g \in \mathscr{G}$ generate $\mathscr{B}_{\mathscr{E}}$, it follows from Lemma 2.1 that $g^{t}$ generate $\mathscr{B}_{t}$ and, by the Multiplicative Systems Theorem,

$$
P\left[Y f\left(X_{t}\right)\right]=P\left[Y f\left(\tilde{X}_{t}\right)\right] \quad \text { for all } f \in \mathscr{B}_{t} .
$$

Therefore

$$
P\left[f\left(X_{t}\right)^{2}\right]=P\left[f\left(\tilde{X}_{t}\right)^{2}\right]=P\left[f\left(X_{t}\right) f\left(\tilde{X}_{t}\right)\right]
$$

which implies 2.3.D.

Clearly, 2.3.E is an implication of 2.3.C and 2.3.D.

2.4. Proof of Theorem 1.1. Let $p$ be a regular transition function and let $\mathscr{Z}$ be a corresponding well-defined regular Markov process. Clearly, 1.4.A holds for 
the algebra $\mathscr{G}$ generated by the family $\mathscr{Q}$ introduced in 1.3.B. It follows from 2.2.B that every $P \in \mathscr{K}_{\alpha}(\mathscr{X})$ has the form

$$
P=\int \mu(d x) P_{\alpha, x}
$$

and therefore $\mathscr{K}_{\alpha e}^{1}(\mathscr{Z}) \subset \mathscr{N}_{\alpha}(\mathscr{Z})$ which implies 1.4.B.

Suppose that $p$ satisfies conditions 1.4.A and B. By 1.4.B, $E_{t}^{\circ}=E_{t}$. Consider $\mathscr{Z}, \tilde{\mathscr{X}}$ and $\tilde{p}$ defined in Theorem 2.1. By 1.4.B, 2.3.A and 1.1.D, $E_{t}=\tilde{E}_{t}$, and by 2.3.D, $E, \tilde{\mathscr{X}}=\left(\tilde{X}_{t}, \mathscr{F}(\Delta), P_{s, x}\right)$ is a Markov process with the transition function $p$. It follows from 2.3.A that $\mathscr{K}_{r}^{1}(\mathscr{X})=\mathscr{K}_{r}^{1}(\tilde{\mathscr{X}})$ and $\tilde{\mathscr{X}}$ is well defined since $\mathscr{Z}$ is well defined. By 2.3.F, C $\mathscr{Z}$ satisfies conditions 1.3.A, C. We construct, on the same space $\Omega$, a Markov process $\hat{\mathscr{X}}=\left(\hat{X}_{t}, \hat{\mathscr{F}}(\Delta), P_{r, x}\right)$ which satisfies, in addition to 1.3.A and $\mathrm{C}$, condition 1.3.B.

To this end, let $\mathscr{W}=\left\{F_{1}(r, x), \ldots, F_{n}(r, x), \ldots\right\}$ be the family in Lemma 2.1 enumerated in an arbitrary order. Formula

$$
Y_{r}=\left\{F_{1}\left(r, \tilde{X}_{r}\right), \ldots, F_{n}\left(r, \tilde{X}_{r}\right), \ldots\right\}
$$

defines a stochastic process in the space $[0,1]^{\infty}$ which we consider as a compact metric space with the distance $d(x, y)=\sum_{1}^{\infty} 2^{-n}\left|x_{n}-y_{n}\right|$. Denote by $D(\Delta)$ the set of mappings from $\Delta$ to $[0,1]^{\infty}$ which are right continuous with left limits. Put $\omega \in A(\Delta)$ if $Y_{t}(\omega), t \in \Delta$, belongs to $D(\Delta)$. We claim that, for every $\omega \in A[r, \infty)$ there exists $\omega^{\prime} \in A(\mathbf{R})$ such that $\omega(t)=\omega^{\prime}(t)$ for all $t \geq r$. Indeed, $P_{x, s}(A[s, \infty))=1$ and therefore $A(\Delta)$ is not empty for any $\Delta$. Let $\omega_{n} \in A\left(\Delta_{n}\right)$ where $\Delta_{n}=[r-n, r-n+1), n=1,2, \ldots$. Put $\omega^{\prime}(t)=\omega(t)$ for $t \geq r$ and $\omega^{\prime}(t)=\omega_{n}(t)$ for $t \in \Delta_{n}$. By 2.3.B, $Y_{t} \in \mathscr{F}\left(\Delta_{n}\right)$ for $t \in \Delta_{n}$; therefore $Y_{t}\left(\omega^{\prime}\right)=Y_{t}\left(\omega_{n}\right)$ for $t \in \Delta_{n}$ and $\omega^{\prime} \in A\left(\Delta_{n}\right)$. For similar reasons, $\omega^{\prime} \in A[r, \infty)$. Hence $\omega^{\prime \prime} \in A(\mathbf{R})$.

Suppose $B \in \mathscr{F}_{>r}$ contains $A(\mathbf{R})$. We claim that $B \supset A[r, \infty)$. Indeed, if $\omega \in A[r, \infty)$, then there exists $\omega^{\prime} \in A(\mathbf{R}) \subset B$ such that $\omega^{\prime}(t)=\omega(t)$ for all $t \geq r$. There exists a countable set $\Lambda \subset(r, \infty)$ such that $B \in \mathscr{F}(\Lambda)$, and $\omega \in B$ because $\omega \in B$ because $\omega^{\prime} \in B$ and $\omega^{\prime}(t)=\omega(t)$ for $t \in \Lambda$. We conclude that, for every $P \in \mathscr{K}_{r}^{1}(\tilde{\mathscr{X}}), P(B) \geq P A[r, \infty)=1$.

Since $A(\mathbf{R})$ has the outer measure 1 relative to $P_{r, x}$, measures $P_{r, x}$ can be continued to the $\sigma$-algebra $\hat{\mathscr{F}}(\mathbf{R})$ generated by $\mathscr{F}(\mathbf{R})$ and $A(\mathbf{R})$. We denote by $\hat{\mathscr{F}}(\Delta)$ the minimal $\sigma$-algebra which contains $\mathscr{F}(\Delta)$ and $A(\mathbf{R})$, we choose an arbitrary element $\omega^{\circ}$ of $A(\mathbf{R})$ and we put

$$
\begin{aligned}
\hat{X}_{t}(\omega) & =\tilde{X}_{t}(\omega) \quad \text { for } \omega \in A(\mathbf{R}), \\
& =\tilde{X}_{t}\left(\omega^{\circ}\right) \quad \text { for } \omega \notin A(\mathbf{R}) .
\end{aligned}
$$

Obviously $\hat{\mathscr{X}}=\left(\hat{X}_{t}, \hat{\mathscr{F}}(\Delta), P_{r, x}\right)$ is a well-defined regular process with the transition function $p$. 


\section{Proof of Theorem 1.2}

3.1. First, we prove Theorem 1.1 for the Dawson-Watanabe supertransition function. The case of the Fleming-Viot supertransition function is much simpler and it will be discussed at the end of the section.

Lemma 3.1. Let $\mathscr{P}$ be the Dawson-Watanabe supertransition function over $p$. Then $\mathscr{P}$ is a Borel transition function. If $p$ separates states and satisfies condition 1.4.A, then the same is true for $\mathscr{P}$.

Proof. Formula

$$
u=\langle 1, \nu\rangle, \quad \nu^{*}(d x)=\nu(d x) /\langle 1, \nu\rangle
$$

defines a one-to-one mapping $\psi$ from $\mathscr{M}_{t} \backslash\{0\}$ onto $(0, \infty) \times \mathscr{M}_{t}^{1}$. Clearly, $\psi$ is measurable and, by 2.1.A, it is an isomorphism. Therefore, $\left(\mathscr{M}_{t}, \mathscr{B}_{\mathscr{M}_{t}}\right)$ is a standard Borel space.

Let $\mathscr{P}(r, \mu ; t, C)=\mathscr{P}(r, \nu ; t, C)$ for all $t, C$. Then, by $(1.8),\left\langle T_{t}^{r} g^{t}, \mu\right\rangle=$ $\left\langle T_{t}^{r} g^{t}, \nu\right\rangle$ for all $g \in \mathscr{G}$. Hence $\left\langle g^{r}, \mu\right\rangle=\left\langle g^{r}, \nu\right\rangle$. By Lemma 2.1, $g^{r}$ generate $\mathscr{B}_{r}$, and by the Multiplicative Systems Theorem, $\mu=\nu$.

Suppose that 1.4.A is satisfied for $p$. Denote by $\mathfrak{G}$ the minimal linear space which contains all the functions

$$
G^{t}(\nu)=e^{-\left\langle g^{t}, \nu\right\rangle}, \quad g \in \mathscr{G}, g \geq 0 .
$$

Since $\mathscr{G}$ is a linear space, $\mathfrak{G}$ is an algebra. By (1.7),

$$
\int \mathscr{P}(r, \mu ; t, d \nu) G^{t}(\nu)=\exp \left\{-\left\langle V_{t}^{r} g^{t}, \mu\right\rangle\right\} .
$$

By (1.6), $V_{t}^{r} g^{t} \rightarrow g^{r}$ as $t \downarrow r$ and therefore 1.4.A is satisfied for $\mathscr{P}$.

3.2. Lemma 3.2. Suppose that $\mathscr{X}=\left(X_{t}, \mathscr{F}(\Delta), P_{r, \mu}\right)$ is a Markov process with transition function $\mathscr{P}$. If $P \in \mathscr{K}_{\alpha e}^{1}(\mathscr{X})$, then $P\left\langle 1, X_{t}\right\rangle\langle\infty$ for all $t>\alpha$.

Proof. Equation (1.6) can be solved explicitly if $f=\lambda$ is a constant, namely,

$$
V_{t}^{r} \lambda=\lambda\left[1+\frac{\gamma}{2} \lambda(t-r)\right]^{-1} \text {. }
$$

Suppose that $r \downarrow \alpha$ along a denumerable set. Then, $P$-a.s.,

$$
P_{r, X_{r}} \exp \left\{-\lambda\left\langle 1, X_{t}\right\rangle\right\} \rightarrow P \exp \left\{-\lambda\left\langle 1, X_{t}\right\rangle\right\}
$$

and, by (1.7) and (3.1),

$$
\lambda\left[1+\frac{\gamma}{2} \lambda(t-r)\right]^{-1}\left\langle 1, X_{r}\right\rangle \rightarrow-\log P \exp \left\{-\lambda\left\langle 1, X_{t}\right\rangle\right\}<\infty .
$$

Hence $\left\langle 1, X_{r}\right\rangle$ tends, $P$-a.s., to a finite limit $c$ and

$$
P \exp \left\{-\lambda\left\langle 1, X_{t}\right\rangle\right\}=\exp \left\{-c \lambda\left[1+\frac{\gamma}{2} \lambda(t-r)\right]^{-1}\right\} .
$$

By taking the derivatives with respect to $\lambda$ at 0 , we get $P\left\langle 1, X_{t}\right\rangle=c$. 
3.3. Theorem 3.1. Let $\mathscr{P}$ be the Dawson-Watanabe supertransition function over $p$. Formula

$$
\kappa_{t}(B)=\int K_{t}(d \nu) \nu(B)
$$

determines a one-to-one mapping $\pi$ from $\mathscr{K}_{\alpha e}^{1}(\mathscr{P})$ onto $\mathscr{K}_{\alpha}(p)$, and the inverse mapping l: $\mathscr{K}_{\alpha}(p) \rightarrow \mathscr{K}_{\alpha e}^{1}(\mathscr{P})$ is given by the formula

$$
\int K_{t}(d \nu) e^{-\langle f, \nu\rangle}=\exp \left\{-S_{t}^{\alpha}(f, \kappa)\right\}
$$

where $f$ is an arbitrary positive $\kappa_{t}$-integrable function and

$$
S_{t}^{\alpha}(f, \kappa)=\left\langle f, \kappa_{t}\right\rangle-\frac{\gamma}{2} \int_{\alpha}^{t}\left\langle\left(V_{t}^{s} f\right)^{2}, \kappa_{s}\right\rangle d s .
$$

Proof. It follows from (1.8) that $\kappa_{r} T_{t}^{r}=\kappa_{t}$ for all $r<t \in \mathbf{R}$. By Lemma 3.2 , the measures $\kappa_{t}$ are finite and therefore $\kappa \in \mathscr{K}_{\alpha}(p)$. By Theorem 1.2 in [D6], $\pi$ is one-to-one mapping from $\mathscr{K}_{\alpha e}^{1}(\mathscr{P})$ onto its image $\mathbf{D}_{\alpha}$. Moreover, by (1.39) in the same paper, $\mathbf{D}_{\alpha} \supset \mathscr{K}_{\alpha}(p)$. Thus $\mathbf{D}_{\alpha}=\mathscr{K}_{\alpha}(p)$. The rest of Theorem 3.1 follows immediately from Theorem 1.2 in [D6].

3.4. Lemma 3.3. Suppose that $\mathscr{P}$ separates states. Then condition 1.4.B holds for $\mathscr{P}$ if and only if it holds for $p$.

Proof. Let $\mu \in \mathscr{M}_{\alpha}$. By (1.8), if

$$
K_{t}(C)=\mathscr{P}(\alpha, \mu ; t, C) \text { for } t>\alpha, C \in \mathscr{B}_{\mathscr{M}_{t}},
$$

then $\kappa=\pi(K)$ is given by the formula

$$
\kappa_{t}(B)=\int \mu(d x) p(\alpha, x ; t, B) \text { for } t>\alpha, B \in \mathscr{B}_{t} .
$$

Since $l$ and $\pi$ are inverse, $K=l(\kappa)$ is given by (3.5) if $\kappa$ is defined by (3.6).

Suppose that $\mathscr{K}_{\alpha e}^{1}(\mathscr{P})=\mathscr{N}_{\alpha}(\mathscr{P})$. If $\kappa \in \mathscr{K}_{\alpha e}^{1}(p)$, then $K=l(\kappa) \in \mathscr{K}_{\alpha e}(\mathscr{P}) \subset$ $\mathscr{N}_{\alpha}(\mathscr{P})$ and therefore $\kappa=\pi(K)$ has the form (3.6). Since $\kappa$ is extremal, $\mu$ is concentrated at one point and $\kappa \in \mathscr{N}_{\alpha}(p)$. Since $\mathscr{P}$ separate states, every $\kappa \in \mathscr{N}_{\alpha}(p)$ is extremal.

For every $\kappa \in \mathscr{K}_{\alpha}(p)$, there exists a finite measure $\gamma$ on $Q=\{\tilde{\kappa}: \tilde{\kappa} \in$ $\left.\mathscr{K}_{\alpha e}(p),\left\langle 1, \tilde{\kappa}_{t}\right\rangle=1\right\}$ such that

$$
\kappa=\int \tilde{\kappa} \gamma(d \tilde{\kappa})
$$

(see [D2 or D4], note that $\left\langle 1, \tilde{\kappa}_{t}\right\rangle$ does not depend on $t$ ). By 1.1.D the mapping $\imath: E_{\alpha} \rightarrow \mathscr{N}_{\alpha}(p)$ defined by (1.5) is an isomorphism. Suppose that $\mathscr{K}_{\alpha e}^{1}(p)=$ $\mathscr{N}_{\alpha}(p)$. Change of variables $\tilde{\kappa}=l(x), x \in E_{\alpha}$ in (3.7) yields

$$
\kappa=\int l(x) \mu(d x)
$$

where $\mu(d x)=\gamma\{l(d x)\}$. Clearly (3.8) is equivalent to (3.6). Thus every $\kappa \in \mathscr{K}_{\alpha}(p)$ has the form (3.6), and every $K \in \mathscr{K}_{\alpha e}(\mathscr{P})$ can be represented by 
the formula (3.5), i.e., $\mathscr{K}_{\alpha e}^{1}(\mathscr{P}) \subset \mathscr{N}_{\alpha}(\mathscr{P})$. The inclusion $\mathscr{N}_{\alpha}(\mathscr{P}) \subset \mathscr{K}_{\alpha e}^{1}(\mathscr{P})$ follows from Theorem 3.1 because $K=l(\kappa)$ if $K$ and $\kappa$ are given (3.5) and (3.6).

Theorem 1.2 follows from Theorem 1.1 and Lemmas 3.1 through 3.3.

3.5. In the case when $\mathscr{P}$ is the Fleming-Viot supertransition function over $p$ Theorem 1.2 follows, in a similar way, from Theorem 1.1 and Theorem 4.2 in [D6].

\section{Concluding Remarks}

4.1. Concept of a right process-in homogeneous setting-is due to Meyer. Getoor [G] has investigated it under weaker assumptions. A recent presentation of the homogeneous theory can be found in [DM or S]. Regular processesboth, in homogeneous and nonhomogeneous cases-have been studied in [D2, $\mathrm{D} 3$ and $\mathrm{Ku}$.

Let us discuss briefly various forms of the definition of such processes.

A positive measurable function $h^{t}(x)$ is called a p-exit rule if $T_{t}^{r} h^{t} \leq h^{r}$ and $T_{t}^{r} h^{t} \uparrow h^{r}$ as $t \downarrow r$. We denote $\mathscr{H}(p)$ the set of all such functions. A Markov process $\mathscr{X}$, subject to conditions 1.3 . B, C is regular if and only if:

4.1.A. For every $h \in \mathscr{H}(p)$ and every $P \in \mathscr{K}_{r}^{1}(\mathscr{Z}), h^{t}\left(X_{t}\right)$ is, $P$-a.s., right continuous in $t$ on $[r, \infty)$.

Suppose that a set $\mathscr{Q}$ of positive functions has the following properties:

(i) If, $q_{1}, q_{2} \in \mathscr{Q}$ then $c_{1} q_{1}+c_{2} q_{2} \in \mathscr{Q}$ for all constants $c_{1}, c_{2} \geq 0$.

(ii) If $q_{n} \in \mathscr{Q}$ and $q_{n} \uparrow q$, then $q \in \mathscr{Q}$.

Then we say that $\mathscr{Q}$ is a closed cone. We denote by $c(A)$ the minimal closed cone which contains $A$. The class of regular process can be described by conditions 1.3.B, C and 4.1.A with $\mathscr{H}(p)$ replaced by any $A$ such that $c(A)=\mathscr{H}(p)$.

A transition function $p$ is stationary if $\left(E_{t}, \mathscr{B}_{t}\right)=(E, \mathscr{B})$ does not depend on $t$ and if $p(r, x ; t, B)=p_{t-r}(x, B)$ depends only on the difference $t-r$. If $p_{t}(x, B)$ is $\mathscr{B}_{\mathbf{R}} \times \mathscr{B}$-measurable in $t, x$ then $\left(\mathscr{E}, \mathscr{B}_{\mathscr{E}}\right)=\left(\mathbf{R}_{+}, \mathscr{B}_{\mathbf{R}_{+}}\right) \times(E, \mathscr{B})$ and 1.5.A is satisfied.

Condition 1.4.A is equivalent to:

4.1.B. The $\sigma$-algebra $\mathscr{B}$ is generated by an algebra $\mathscr{G}$ of bounded functions $g$ such that $T_{t} g(x) \rightarrow g(x)$ for all $x$ as $t \downarrow 0$.

If $\mathscr{B}$ is the Borel $\sigma$-algebra in a metric space, then the role of $\mathscr{G}$ is usually played by the set of all bounded continuous functions.

Let $\lambda \geq 0$. A positive $\mathscr{B}$-measurable function $h$ is called $\lambda$-excessive if $e^{-\lambda t} T_{t} f \leq f$ and $e^{-\lambda t} T_{t} f \uparrow f$ as $t \downarrow 0$. The class of regular processes can be defined by 1.3.B, $\mathrm{C}$ and:

4.1.C. For every $P \in \mathscr{K}_{0}^{1}(\mathscr{X})$ and every $\lambda$-excessive function $f, f\left(X_{t}\right)$ is $P$-a.s., right continuous in $t$ for $t \geq 0$.

4.2. Each of conditions 1.3.A, 4.1.A and 4.1.C, with $\mathscr{K}_{r}^{1}(\mathscr{X})$ replaced by $\mathscr{N}_{r}(\mathscr{X})$, determines, in combination with 1.3.B, C the class of right processes. 
A homogeneous right process can be nonregular. However, the corresponding Ray process when restricted to the set of its nonbranching points is regular.

The regularization of $\mathscr{X}$ used in $\S 2$ is close, in spirit, to the Ray-Knight compactification. However we work with transition functions rather than resolvents and we avoid to specify any topology in state spaces. The branching points defined in $\S 2.3$, correspond to nondegrenerate branching points in theory of Ray processes.

4.3. The Dawson-Watanabe supertransition function can be defined in certain spaces of $\sigma$-finite measures.

Suppose that $h^{t}(x)$ is a strictly positive finite exit rule for $p$ and denote by $\mathscr{M}_{h t}$ the set of all measures $\nu$ on $\left(E_{t}, \mathscr{B}_{t}\right)$ such that $\left\langle h^{t}, \nu\right\rangle<\infty$. Let $\mathscr{B}_{h t}$ stand for the natural $\sigma$-algebra in $\mathscr{M}_{h t}$. (Note that $\left(\mathscr{M}_{h t}, \mathscr{B}_{h t}\right)=\left(\mathscr{M}_{t}, \mathscr{B}_{\mathscr{M}_{t}}\right)$ if $h=1$.) By Theorem 3.1 in [D6], there exists a unique transition function $\mathscr{P}$ in $\left(\mathscr{M}_{h t}, \mathscr{B}_{h t}\right)$ such that (1.6) and (1.7) hold for all positive (not only bounded) $\mathscr{B}_{t}$-measurable functions $f$.

For a general $h$, we are able to prove only a weaker version of Theorem 1.2.

Denote by $\hat{\mathscr{K}}_{\alpha}(\mathscr{X})$ the set of all measures on $\mathscr{F}_{>\alpha}$ subject to condition (1.3) such that, for every $t>\alpha, P\left\{X_{t} \in B\right\}=\kappa_{t}(B)$ is a $\sigma$-finite measure. Obviously, $\mathscr{N}_{\alpha}(\mathscr{X}) \subset \mathscr{K}_{\alpha}^{1}(\mathscr{X}) \subset \hat{\mathscr{K}}_{\alpha}(\mathscr{X})$.

Theorem 4.1. Let $p$ be a Borel transition function which separates states and let $\mathscr{P}$ be the corresponding Dawson-Watanabe supertransition function in $\left(E_{t h}, \mathscr{B}_{t h}\right)$. If $p$ is regular, then $\mathscr{P}$ is right.

The proof of Theorem 4.1 is based on Theorem 1.2 in [D6] and a modification of Theorem 1.1.

Theorem 4.2. Let $\mathscr{K}_{\alpha e}^{1}(p, h)$ stand for the set of all $\kappa \in \mathscr{K}_{\alpha e}^{1}(p)$ such that

$$
\sup _{t>\alpha}\left\langle h^{t}, \kappa_{t}\right\rangle<\infty \text {. }
$$

A transition function $p$ is right if 1.4.A holds and if, for every $\alpha \in \mathbf{R}, \mathscr{K}_{\alpha e}^{1}(p, h)=$ $\mathscr{N}_{\alpha}(p)$.

Proof. Consider $\mathscr{X}, \tilde{\mathscr{X}}$ and $\tilde{p}$ defined in Theorem 2.1. Under conditions of Theorem 4.2, $E_{t}^{\circ}=E_{t}$ can be identified with $\mathscr{K}_{t e}^{1}(p, h) \subset \mathscr{K}_{t e}^{1}(\mathscr{P})=\tilde{E}_{t}$ in such a way that $x \in E_{t}$ if and only if

$$
\sup _{s>t} \tilde{P}_{t, x} h^{s}\left(X_{s}\right)<\infty .
$$

Since $P_{s, x} h^{u}\left(X_{u}\right) \leq h^{s}(x)$, it follows from 2.3.F that, for every $r \in \mathbf{R}, x \in E_{r}$,

$$
\sup _{r<i<u} P_{t, \dot{X}_{t}} h^{u}\left(X_{u}\right) \leq \sup _{s>r, s \in \Lambda} h^{s}\left(X_{s}\right), \quad P_{r, x} \text {-a.s. }
$$

The right side is finite $P_{r, x}$-a.s. because $\left\{h^{s}\left(X_{s}\right), \mathscr{F}_{\leq s}, P_{r, x}\right\}$ is a positive supermartingale on $(r, \infty)$ and $P_{r, x} h^{s}\left(X_{s}\right) \leq h^{r}(x)<\infty$ for all $s>r$. Therefore $\tilde{X}_{t} \in E_{t}$ for all $t>r P_{r, x}$-a.s. 
Choose an arbitrary point $c_{t}$ in $E_{t}$ and put

$$
X_{t}^{\prime}(\omega)=\tilde{X}_{t}(\omega) \quad \text { if } \tilde{X}_{t}(\omega) \in E_{t}, \quad X_{t}^{\prime}(\omega)=c_{t} \quad \text { if } \tilde{X}_{t}(\omega) \notin E_{t} .
$$

Clearly $\mathscr{X}^{\prime}=\left(X_{t}^{\prime}, \mathscr{F}(\Delta), P_{r, x}\right)$ is a Markov process in $\left(E_{t}, \mathscr{B}_{t}\right)$ with transition function $p$. By 2.3.A, $\mathscr{K}_{r}^{1}(\mathscr{X})=\left\{P_{r, \mu}, \mu \in \mathfrak{M}_{r}^{r}\right\}$ and therefore $\mathscr{X}^{\prime}$ is right. (Conditions 1.3.B, C can be checked as in proof of Theorem 1.1.)

Acknowledgement. I want to thank P. Fitzsimmons and R. K. Getoor for valuable comments which lead to significant improvements of the first version of the paper and, in particular, clarified the relation between regular and right processes.

\section{REFERENCES}

[Da] D. A. Dawson, Stochastic evolution equations and related measure processes, J. Multivariate Anal. 3 (1975), 1-52.

[DH] D. A. Dawson and K. J. Hochberg, Wandering random measures in the Fleming-Viot model, Ann. Probab. 10 (1982), 554-580.

[DM] C. Dellacherie and P. -A. Meyer, Probabilités et potentiel, Chapitres XII à XVI, Hermann, Paris, 1987.

[D1] E. B. Dynkin, Integral representation of excessive measures and excessive functions, Uspekhi Mat. Nauk 27 (1972), no. 1, 43-80. Reprinted in London Math. Soc. Lecture Note Series 54, Cambridge Univ. Press, London and New York, 1982, pp. 145-186.

[D2] _ Regular Markov processes, Uspekhi Mat. Nauk 28 (1973), no. 2, 35-64. Reprinted in London Math. Soc. Lecture Note Series 54, Cambridge Univ. Press, London and New York, 1982, pp. 187-218.

[D3] __ Markov representations of stochastic systems, Uspekhi Mat. Nauk 30 (1975), no. 1, 6199. Reprinted in London Math. Soc. Lecture Note Series 54, Cambridge Univ. Press, London and New York, 1982, pp. 219-258.

[D4] _ Sufficient statistics and extreme points, Ann. Probab. 6 (1978), 706-730. Reprinted in London Math. Soc. Lecture Note Series 54, Cambridge Univ. Press, London and New York, 1982, pp. 259-284.

[D5] _ Superprocesses and their linear additive functionals, Trans. Amer. Math. Soc. (to appear).

[D6] __. Three classes of infinite dimensional diffusions, J. Funct. Anal. (to appear).

[FV] W. H. Fleming and M. Viot, Some measure-valued Markov processes in population genetics theory, Indiana Univ. Math. J. 28 (1979), 817-843.

[G] R. K. Getoor, Markov processes Ray processes and right processes, Lecture Notes in Math., vol. 440, Springer, Berlin and New York, 1975.

[K] K. Kuratowski, Topology, 2 vols., Academic Press, New York, 1967-69.

[Ku] S. E. Kuznetsov, Nonhomogeneous Markov processes, J. Soviet Math. 25 (1984), 1380-1498.

[S] M. J. Sharpe, General theory of Markov processes (forthcoming).

[W] S. Watanabe, A limit theorem of branching processes and continuous state branching processes, J. Math. Kyoto Univ. 8 (1968), 141-167.

Department of Mathematics, White Hall, Cornell University, Ithaca, New York $14853-7901$ 